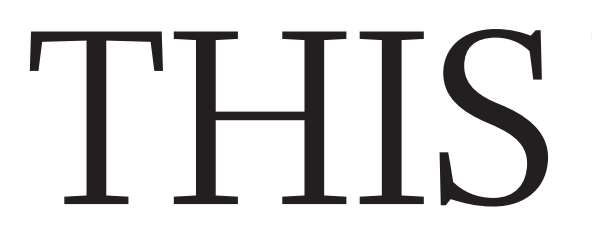

EDITORIALS down on scientific controversy $\mathbf{p . 4 2 6}$
STEM CELLS Curtain comes

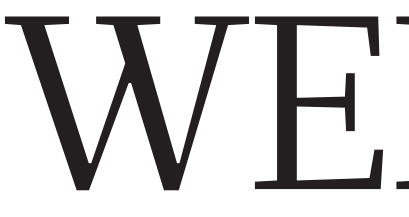

WORLD VIEW End the unfair racket of academic jobs for the boys $\mathbf{p . 4 2 7}$
DECEPTION Orchid shape and smell fools amorous wasps p.429

\title{
In the name of beauty
}

\section{The ugly truth is that the plastic microbeads found in many skin scrubs and other personal-care products are a serious pollutant of the marine environment. They should be phased out rapidly.}

A beautiful woman comes into focus. What makes her skin glow so? Why, she says, she uses Aveeno's Positively Radiant skinbrightening daily scrub for "naturally beautiful results".

What is not clear from this advertisement is that the "gentle exfoliators" in the product promoted by Jennifer Aniston are minuscule beads of plastic. When Aniston, or those she inspires to follow her, rinse the scrub down the drain, many of the beads end up in the sea, where they will persist indefinitely. This is unnecessary, damaging and must stop.

Others agree, and the face scrub, along with hundreds of other products, including toothpastes, may not be long for this world. On 10 September, the California Legislature sent a bill (AB 888) to the state's governor, Jerry Brown, that would ban the inclusion of spheres of polyethylene, polypropylene and other plastics less than 5 millimetres across in personal-care products after 2020 .

If signed into law, the bill will prevent trillions of plastic beads from being rinsed down the drain. Not all of these make it to the sea - wastewater treatment plants can sift out $90 \%$ of them - but the problems caused by the remaining millions are considerable. (Meanwhile, beads trapped in 'sludge' at the plants do not disappear. Plenty are sprayed on crops, from where they escape to rivers and lakes.)

In a paper published on 3 September, aquatic-health researcher Chelsea Rochman at the University of California, Davis, and her colleagues estimate that 8 trillion microbeads per day are emitted into aquatic habitats in the United States alone (C. M. Rochman et al. Environ. Sci. Technol. http://doi.org/7sw; 2015).

The beads are more pernicious than mere litter. Roughly the size of many plankton species, they are eaten by marine creatures. One study in 2014 saw them consumed by several taxa of zooplankton, including mysid shrimps, copepods, rotifers and ciliates (O. Setälä et al. Environ. Pollut. 185, 77-83; 2014). Some of these are then eaten by larger creatures, and toxic chemicals in the plastics, as well as other toxic chemicals that adhere to plastic particles, accumulate in fish - which might end up on our dinner tables.

California would not be the first place to pass a microbead ban, but as the world's seventh- or eighth-largest economy, its move would carry weight. Just as in automotive-fuel efficiency standards or flammability requirements on furniture, where California goes, other places in the United States and elsewhere follow. The California bill is also stronger than many before it. It does not include a common loophole allowing for the use of 'biodegradable' beads - which are unlikely to truly degrade anywhere except in an industrial composter.

California legislators have made the right call, but the phase-out period is too long. No luminous complexion is worth the wholesale pollution of Earth's oceans. Consumer-goods giant Unilever says that it has already removed microbeads from all of its scrubs and washes. And there are plenty of well-tested alternative exfoliants, including nut shells, sand and sugar. So why wait five years to stop polluting?
While bans and phase-outs slowly take effect, the Beat the Microbead campaign, funded by Dutch non-governmental organizations the Plastic Soup Foundation and the North Sea Foundation, has created an app for consumers who want to avoid contributing to the problem. A few clicks can confirm whether the tempting scrub in the pharmacy aisle contains the beads. This is helpful in the short term, but ultimately the onus of responsibility should not be on the consumer.

\section{"No luminous complexion is worth the wholesale pollution of Earth's oceans.'}

Microbeads are not the only source of microplastic in the oceans. Tiny plastic pellets used in making plastic items spill into the sea; plastic bags and bottles break down over time. On almost any beach on Earth, the sand carries tiny, bright grains of plastic.

And macroplastics remain a serious problem. A study published last month estimated that around $90 \%$ of seabirds have plastic in their bellies (C. Wilcox et al. Proc. Natl Acad. Sci. USA http://doi.org/7dv; 2015). Some birds mistake shopping bags for jellyfish; others confuse cigarette lighters and pen caps with prey and fly home to feed them to their chicks.

The consequences of this ubiquitous plastic for marine species, marine ecosystems and human health remain areas of active research. But the public and policymakers need not wait for detailed results before taking action. Banning microbeads will not solve the plasticpollution problem, but it is an easy start. Jennifer Aniston and the millions of other people who wash their faces with plastic can still look radiant without feeding their skincare regime to copepods. The alternative is to forever blush with shame.

\section{Power play}

\section{The replacement of mitochondria does not signal ethical problems.}

$\mathrm{G}$ alloping advances in genetic and stem-cell technologies raise the inviting prospect that some devastating diseases could be treated. Conditions caused by natural mutations might be avoided by judicious genome tinkering to set things right for the next generations. But 'inviting' does not always mean 'advisable'.

The United Kingdom last week released new draft guidelines for one such treatment - mitochondrial replacement (see go.nature. com/thcouy). The guidelines are scheduled to come into force next month, when clinics in Britain will be allowed to offer the treatment. Not everybody agrees that this inviting idea is advisable. As such, it is timely to consider the ethical and technical matters at stake. 